\title{
Adaptive Scheme for suppressing unnecessary Hello messages used for Neighbor Discovery in MANET routing protocols
}

\author{
Harpreet Kaur1 ${ }^{1}$ Er.Jasmeet Brar² (Guide) \\ ${ }^{1} J C D M$ College of Engineering Sirsa, India \\ ${ }^{2} J C D M$ College of Engineering Sirsa, India \\ harpreetk514@gmail.com \\ brar7474@gmail.com
}

\begin{abstract}
Mobile ad-hoc networks are widely used these days as in smart phones but energy efficiency is a major issue when discovering neighbor nodes to maintain connectivity as periodically exchanging hello messages are used for it. Overhead goes high due to neighbor discovery messages in the MANET routing protocols, such as in AODV, DYMO. In an attempt to enhance the scheme, this paper proposes a new approach to solve the problem by reducing unnecessary hello messages. In this paper we consider MANET under random waypoint model and investigated the relationship between the hello interval and event intervals. Finally we evaluate our study through simulations, the proposed scheme reduces the energy consumption and overhead of neighbor discovery processes.
\end{abstract}

Index Terms: - Hello messaging, energy consumption, network overhead, hello interval, mobile ad-hoc network (MANET).

\section{INTRODUCTION}

A Mobile ad-hoc network is a network without infrastructure, so in it every node works as a router[1]. In this network, every node must discover its local neighbors and through those neighbors it will communicate to nodes that are out of its transmission range (multi-hop). The neighbor discovery process periodically broadcasts a HELLO message to find available neighbors. An acknowledgment should be sent in response to the HELLO message within a specific time.

Many Hello messaging schemes focus on figuring out dynamic network topology or discovering live neighbors with an energy saving scheme, which requires all network nodes to continuously exchange Hello messages or beacons while they are awake. In such traditional Hello messaging schemes no start/end condition is described. This can cause unnecessary bandwidth usage and hidden energy consumption if MANET routing protocol (e.g., Ad hoc On-Demand Distance Vector (AODV) , or Dynamic MANET On-demand routing (DYMO) is used, where a new path is discovered through Route Request (RREQ) and Route Response (RREP) packet exchanges. Two commonly used routing strategies are reactive and proactive. Reactive routing protocols creates routes only when they are required (e.g., DSR, AODV). Proactive routing protocols are table driven and utilize table to determine the next hop to reach the destination (DYMO, OLSR, DSDV). In MANETs, any node in a route can be turned off or can move away, which negatively affects route maintenance and this may cause delay in the data dissemination. It is important for a node in MANET to discover live neighbor nodes through hello messaging [3]. Hello messages must be generated and transmitted at each MANET node independently. Two successive transmissions of hello message on the same MANET node must be separated by least hello interval [4].

In conventional hello messaging scheme, the hello messages are sent on the basis of constant hello intervals for hello messages broadcasting to the network for route establishment and maintenance [5] and also no start/end condition is described [3]. Most ad hoc routing protocols may detect broken links using hello messages and the feedback provided to the protocol by MAC (Medium Access Control) layer. At low network load MAC feedback works better than hello messages but when the traffic load on the network increases then due to incorrect link failure decisions throughput decreases. Routing protocols require hello messaging when run over the link layer protocols that do not provide MAC feedback when the next hop is unreachable. In standard AODV using periodic hello messaging, failed links are to be detected before a packet is sent to the next hop. But MAC layer protocols do not provide feedback about the presence of the next hop. Also the consumed energy and the volume of the control traffic is greater when AODV is run over MAC layer protocols [6]. Hence hello messaging is preferred over Link layer feedback mechanism. Giruka and Singhal proposed two approaches for suppressing the hello messages whenever they are not required, an on-demand mechanism and a monitoring activity mechanism. The on-demand mechanism also called hello protocol enables hello protocol only when it is demanded using a hello request-reply mechanism, but because of additional packet exchange before 
communication delay increases. The monitoring activity mechanism also called event based hello protocol and it enables only those nodes to broadcast hello packets that are active, the hello packets are broadcasted based on a threshold called an activity timer [7].

In this paper we investigate the adaptive hello messaging scheme for neighbor discovery, which belongs to the MANET routing protocols. The adaptive hello messaging scheme aims to reduce the unnecessary hello messages by making hello interval proportional to the event interval of a node. First we design the network by taking random network topology and random events are taken and hello messaging scheme used initially by using constant hello interval. Then we enhance the scheme by making hello interval proportional to event interval i.e. as the event will take place the node will start sending hello messages, hence hello messaging scheme made dynamic. Via simulation experiments we evaluate these schemes under different parameter settings of dynamic environment to see their performance in MANETs related to energy efficiency and battery consumption.

The rest of paper is organized as follows: In section II related work is discussed. The proposed method of dynamic hello messaging scheme is presented in section III. In section IV we give the results obtained by applying method to the MANET. Finally section V concludes this paper with some discussions on the future work.

\section{RELATED WORK}

The hello messaging scheme has been investigated extensively in the literature. In [8], an implementation of Ad hoc on demand distance vector (AODV) is utilized to determine the effectiveness of hello messages for determining local connectivity. To increase the effectiveness of the hello messages, the reception characteristics of hello messages should be equal to that of the data packets and for this two must have similar characteristics of rate and size and hence better throughput will result in this case. In [6], the advantages and drawbacks of both the techniques hello messaging and MAC feedback i.e. used to determine link failures in ad hoc routing protocols are addressed. Simulation results showed that MAC feedback works better than hello messages with low network load, but if the network load increases, the incorrect decision about link failure also increases which results in lower throughput. Also when AODV is run over MAC layer protocols, the energy that is consumed and traffic control consumed with the MAC protocols are greater than when they run over MAC IEEE 802.11. In [7], impact of the hello protocols on adhoc networks is discussed. The basic idea behind the proposed scheme is to send hello messages as little as possible, the proposed hello protocols incur less overhead and the network performance increases compared to a periodic hello messaging protocol, while maintaining the accuracy of identical neighbor table. In [9], the relationship between the transmission frequency of hello messages and the sensing timer expiration value is investigated with the network's node mobility. In [10], neighbor discovery is exploited and overhead of neighbor discovery processes is reduced.

However all these schemes address only conventional hello messaging scheme using constant hello interval. But the network topology changes rapidly in MANETs due to random node mobility and for neighbor discovery hello messages are sent after regular intervals i.e. constant hello interval and hence battery consumption increases, energy efficiency decreases.

\section{PROPOSED SCHEME}

In this section we first present our network model and then formulate the adaptive scheme for hello messaging. We consider MANET operating within random network topology and random nodes. Node mobility follows a model, there is several mobility models used in the evaluation of MANET protocols. The random waypoint model is one the most commonly used mobility models for simulations of MANETs. In this model, each node selects a random destination, uniformly distributed within the two-dimensional space [11]. In conventional hello messaging scheme before a packet is sent, status of neighbor nodes should be recognized first so as to recognize if there is a link failure with one of its neighboring nodes. If the node moves to an area where no active nodes are in its neighborhood, then nodes keeps broadcasting hello messages and due to this unnecessarily energy consumption takes place. For this problem hello messages should be suppressed first on the basis of determining value of hello interval. The maximum interval of time between the transmissions of hello messages is HELLO_INTERVAL. The time during which the node should assume that link is currently broken is the time if a node does not receive any packet from that node within the given time i.e.

$$
\mathrm{T}_{\mathrm{fd}}=(\text { ALLOWED_HELLO_LOSS)*(HELLO_INTERVAL). }
$$

By default, ALLOWED_HELLO_LOSS is equal to 2 and HELLO_INTERVAL is equal to $1000 \mathrm{~ms}$ i.e. $1 \mathrm{sec}$ for AODV [12]. Now in the dynamic hello messaging scheme the time for link failure detection $\left(\mathrm{T}_{\mathrm{FDD}}\right)$ is given as:

$$
\mathrm{T}_{\mathrm{fdd}}=(\text { ALLOWED_HELLO_LOSS-.5)*(HELLO_INTERVAL) (2) }
$$


Main objective of our research is reduction of energy consumption and network overhead. We can do this only when hello interval is made proportional to the event interval of a node. If the hello interval is set without considering the event interval of a node, then the risk of sending packet through an unavailable link increases $\left(\mathrm{P}_{\mathrm{fdd}}\right)$. In our research we are considering the scenario of an urban city and Exponential traffic distribution for our research. We assumed the $\mathrm{C}_{\mathrm{DF}}$ of event interval in Fig. 1 and the assumption is considered for the 100 events, where it is analyzed for the time of 1 hour and is given by:

$$
X(\alpha, \beta)=1-e^{-\alpha / \beta}
$$

We consider $\mathrm{X}(\mathrm{a}, \mathrm{b})$ as probability of the total no of outcomes bounded by exponential distribution. We can write this as:

$$
\begin{aligned}
& \mathrm{P}_{\mathrm{fdd}}=1-\mathrm{e}^{-\alpha / \beta} \\
& \ln \left(\mathrm{P}_{\mathrm{fdd}}\right)=\ln \left(1-\mathrm{e}^{-\alpha / \beta}\right) \\
& \ln \left(\mathrm{P}_{\mathrm{fdd}}\right)=\ln (1)-\ln \left(\mathrm{e}^{-\alpha / \beta}\right) \\
& a=-b \ln \left(1-P_{f d d}\right)
\end{aligned}
$$

Here $a$, is in linear relationship with $b$, so the neighboring node to calculate the value of hello interval use $b$, to maximize the value of $\mathrm{T}_{\mathrm{FDD}}$. With the value of $\mathrm{T}_{\mathrm{FDD}}$, we can calculate the value of hello interval by using equation 2 .

The distribution of the packet reception of the event intervals on each node is investigated with 20 nodes. The most of event intervals are less than the default hello interval i.e. $1 \mathrm{sec}$; we investigated the event intervals that are larger than the $1 \mathrm{sec}$, because hello interval will not be modified if value will be less than $1 \mathrm{sec}$. The probability of failure detection of an unavailable link is made proportional to exponential traffic distribution.

\section{EVALUATION}

The network size is fixed to 20 nodes located in a $1000 \mathrm{~m}$ x $1000 \mathrm{~m}$ square. Mobility model chosen is random way point model, in this model nodes chooses a random destination point. In the simulation we concentrated on two performance metrics: network overhead and energy consumption.

We modified AODV and DYMO with the proposed scheme i.e. AODV-AH and DYMO-AH respectively. The default Pfd is set to 20\%. The simulation parameters shown in Table I. are used.

\begin{tabular}{|c|c|}
\hline Parameter & Value \\
\hline Mobility model & Random way point model \\
\hline $\begin{array}{c}\text { Transmission } \\
\text { power }\end{array}$ & $16 \mathrm{dBm}$ \\
\hline Topology size & $1000 \mathrm{mx} 1000 \mathrm{~m}$ \\
\hline No of nodes & 20 \\
\hline Packet size & 210 bytes \\
\hline Simulation time & 30 \\
\hline Data traffic & Exponential \\
\hline Pause time & 0 sec \\
\hline
\end{tabular}

TABLE I. Simulation Parameters

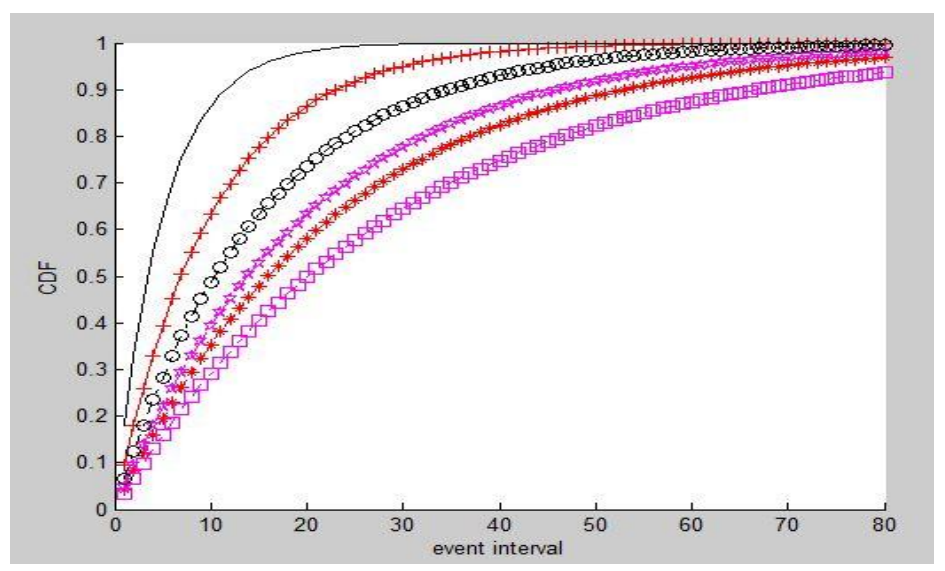

Fig.1. Distribution of event intervals 
Fig. 2 shows the comparison of energy consumption between DYMO and DYMO-AH. Each node is assigned with 1 joule of energy initially. As time goes on increasing DYMO consumes energy quickly due to periodic hello messaging scheme and hence more energy consumption and battery drain will take place. When DYMO$\mathrm{AH}$ is applied, unnesseccary hello messages are suppressed and hence energy consumed is less due to less transmissions and reception of hello messages.

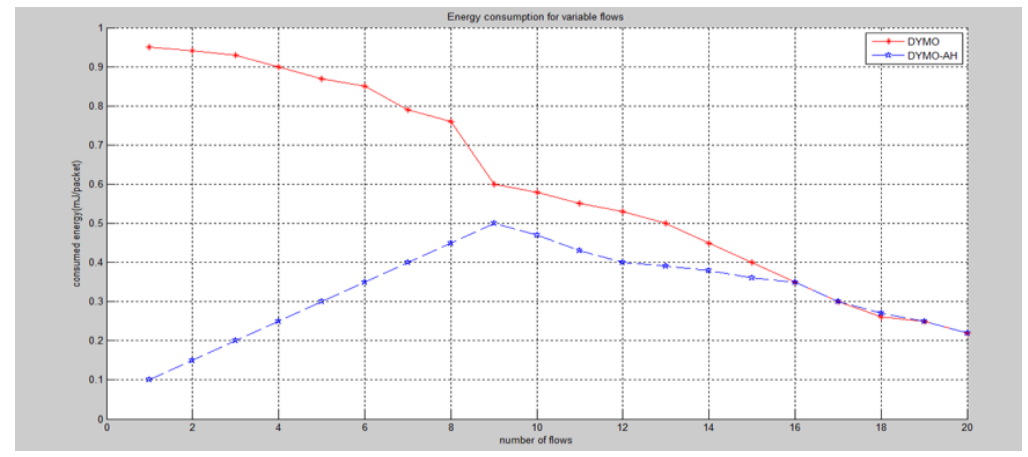

Fig.2. Energy consumption for variable flows

Fig. 3 shows the hello packet overhead. Here number of the hello packets is decreased by as much as half due to the proposed scheme, because as number of nodes will increase, the number of hello packets received by a node will also increase.

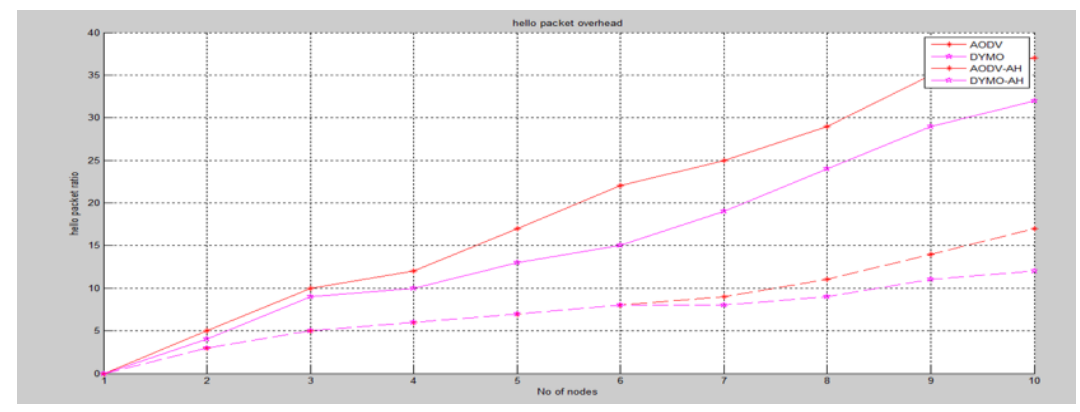

Fig.3.Hello packet overhead

Fig 4.compares the energy consumed by AODV and AODV-AH. Initially each node has 160 joules of energy. Here remaining average energy of all nodes are taken. As number of nodes will increase AODV consumes energy quickly, while AODV-AH due to the proposed scheme will reduce unnecessary hello messages hence, take energy consumption will take more time.

Figure 5 shows the number of nodes that will increase their Hello intervals. The cumulative probability of active nodes that send or receive packets during recent $\mathrm{x}$ seconds $(\mathrm{x}=3,5$, and $10 \mathrm{sec})$ are shown. The number of flows is represented on the $\mathrm{x}$-axis. When there are $10 \mathrm{ftp}$ flows and $\mathrm{x}=3 \mathrm{sec}, 85 \%$ of nodes do not send or receive in the most recent 3 seconds - i.e., there is no need refresh within 3 seconds. This shows that a large portion of nodes use a longer Hello interval than the current default.

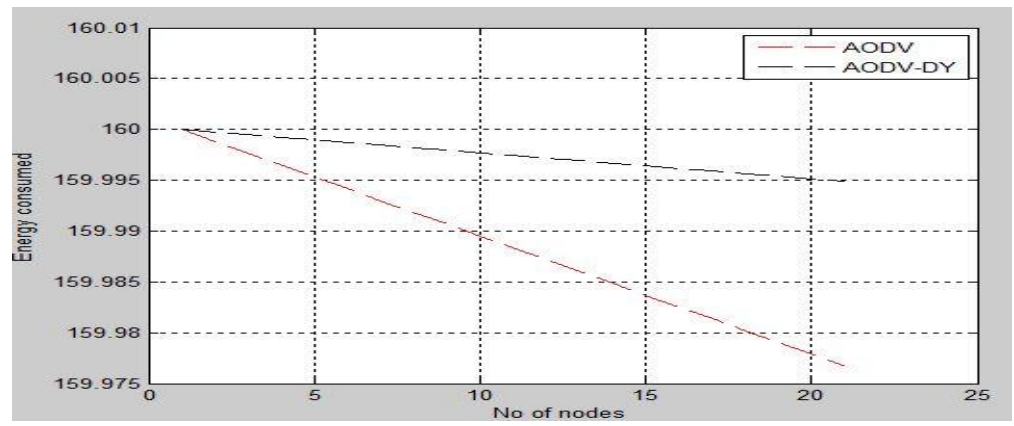

Fig.4. Energy consumption for AODV 


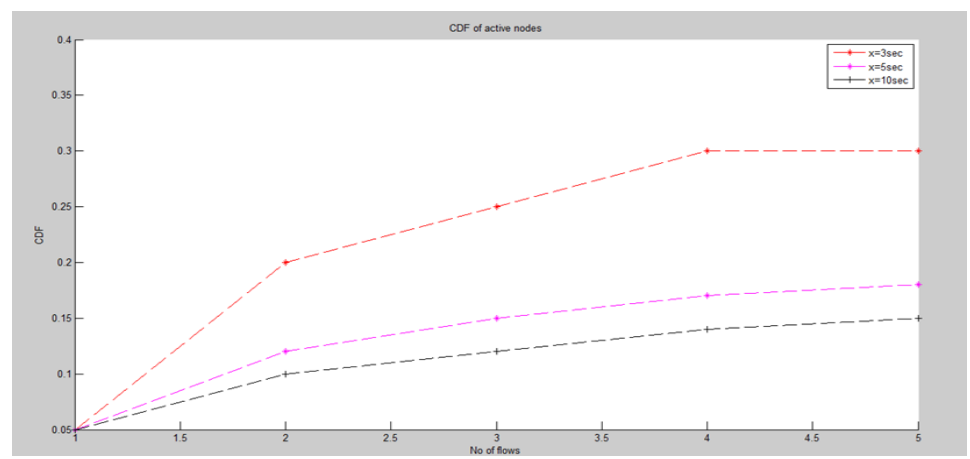

Fig.5. CDF of active nodes

\section{CONCLUSION AND FUTURE WORK}

In this paper, a new approach, called Adaptive Hello Messaging scheme is proposed to solve the problems related to battery consumption and network overhead. The Hello Messaging Scheme aims to reduce unnecessary hello messages while neighbor discovery and also to establish a reliable connection between the source node to the destination node. This is one of the important issues that significantly affect the performance of the MANETs.

As a future work, we can deploy the proposed approach in more scenarios and large scale networks, and we plan to extend our work to optimize the value of the hello intervals by using different optimization techniques.

\section{REFERENCES}

[1] I. Chlamtac, M. Conti, and J. J. Liu. Mobile ad hoc networking: Imperatives and challenges. Ad Hoc Networks, 1(1):13-64, 2003. Cited By (since 1996):376.

[2] S. Corson, J. Macker., "Mobile Ad hoc Networking (MANET): Routing Protocol Performance Issues and Evaluation Considerations," IETF RFC2501, 1999.

[3] Seon Yeong Han, and Dongman Lee "An Adaptive Hello Messaging Scheme for Neighbor Discovery in On-Demand MANET Routing Protocols" IEEE Communications Letters, VOL. 17, NO. 5, MAY 2013.

[4] T. Clausen, C. Dearlove, and J. Dean, "Mobile ad hoc network (MANET) neighborhood discovery protocol (NHDP)," 2010.

[5] David B. Johnson, David A. Maltz, Yih-Chun Hu and Jorjeta G. Jetcheva, "The Dynamic Source Routing for Mobile Ad Hoc Wireless Networks, “ July 2004.

[6] Dimitri Marandin, "Performance Evaluation of Failed Link Detection in Mobile Ad Hoc Networks," 3rd Annual Med-Hoc-Net, 2004.

[7] V. C. Giruka and M. Singhal, "Hello protocols for ad-hoc networks: overhead and accuracy tradeoffs," in Proc. Sixth IEEE International Symposium on a World of Wireless Mobile and Multimedia Networks, pp. 354-361.

[8] Ian D. Chakeres, Elizabeth M. Belding-Royer, "The Utility of Hello Messages for Determining Link Connectivity" in Proc. 2002 Wireless Personal Multimedia Communications, 2002. The 5th International Symposium.

[9] R. Oliveira, M. Luis, L. Bernardo, R. Dinis, and P. Pinto, "The impact of node's mobility on linkdetection based on routing hello messages," in Proc. 2010 IEEE Wireless Communications and Networking Conference, pp. 1-6.

[10] Ehsan Mostajerani, Rafidah Md Noor and Hassan Keshavarz, "A Novel Improved Neighbor Discovery Method for an Intelligent-AODV in Mobile Ad hoc Networks," International Conference of Information and Communication Technology, 2013.

[11] V. D. Tracy Camp, Jeff Boleng, "A survey of mobility models for ad hoc network research," Wireless Communications and Mobile Computing, 2:483-502, 2002.

[12] E. Belding-Royer and S. D. C. Perkins, "Ad hoc on-demand distance vector (AODV) routing," July 2003. 